\title{
Co-culture of Bacillus amyloliquefaciens ACCC1 1060 and Trichoderma asperellum GDFS1009 enhanced pathogen-inhibition and amino acid yield
}

\author{
Qiong $\mathrm{Wu}^{1+}, \mathrm{Mi} \mathrm{Ni}^{2+}, \mathrm{Kai} \mathrm{Dou}^{1 \dagger}{ }^{\dagger}$ Jun Tang ${ }^{2}$, Jianhong Ren ${ }^{3}$, Chuanjin $\mathrm{Yu}^{1}$ and Jie Chen ${ }^{1 *}$
}

\begin{abstract}
Background: Bacillus spp. are a genus of biocontrol bacteria widely used for antibiosis, while Trichoderma spp. are biocontrol fungi that are abundantly explored. In this study, a liquid co-cultivation of these two organisms was tried firstly.

Results and discussion: Through liquid chromatography-mass spectrometry/mass spectrometry (LC-MS/MS), it was discovered that with an inoculation in the ratio of 1.9:1, the antimicrobial effect of the co-cultured fermentation liquor of Bacillus amyloliquefaciens ACCC11060 and Trichoderma asperellum GDFS1009 was found to be significantly higher than that of pure-cultivation. A raise in the synthesis of antimicrobial substances contributed to this significant increase. Additionally, a co-culture with the inoculation of the two organisms in the ratio of 1:1 was found to enhance the production of specific amino acids. This technique could be further explored for either a large scale production of amino acids or could serve as a theoretical base for the generation of certain rare amino acids.
\end{abstract}

Conclusions: This work clearly demonstrated that co-cultivation of B. amyloliquefaciens ACCC11060 and T. asperellum GDFS1009 could produce more specific biocontrol substances and amino acids.

Keywords: Bacillus amyloliquefaciens, Trichoderma asperellum, Liquid co-cultivation, Amino acid, Biocontrol metabolites

\section{Background}

Bacillus spp. exhibit antagonistic effects against a variety of pathogenic fungi, as well as multifarious pathogenic bacteria [1]. They are reported to induce pathogen inhibition by antibiosis through the secretion of many secondary metabolites [2]. Different Bacillus species secrete diverse substances that include surfactins [3], fengycins [4], bacisubin [5] and polyketides [6]. It has been shown that 3-hydroxypropionaldehyde, produced by Bacillus, efficiently inhibits the growth of Alternaria solani,

*Correspondence: jiechen59@sjtu.edu.cn

${ }^{\dagger}$ Qiong Wu, Mi Ni and Kai Dou are co-first authors

1 School of Agriculture and Biology, Shanghai Jiao Tong University,

Shanghai 200240, China

Full list of author information is available at the end of the article
Botrytis cinerea, Fusarium sambucinum, and Pythium sulcatum [7].

Trichoderma spp. are known to exhibit antagonistic effects against at least 18 genera and 29 species of pathogenic fungi. The biocontrol mechanisms of Trichoderma spp. primarily include competition and mycoparasitism, followed by the stimulation of plant resistance and immunity [8]. The most significant antagonistic mechanism of Trichoderma spp. against pathogens is mycoparasitism. This process is accompanied by the secretion of cell wall-degrading enzymes (CWDEs) such as chitinases, glucanase, and proteases which penetrate the pathogen mycelium, absorbing its nutrients and ultimately dissolving the pathogen $[9,10]$. Additionally, Trichoderma spp. are reported 
to induce pathogen inhibition by secreting secondary metabolites such as 6-pentyl- $\alpha$-pyrone [11, 12].

Although both Bacillus and Trichoderma spp. are known to display good biological control, their primary mechanisms for biocontrol are not the same. Despite their capabilities to generate antibacterial secondary metabolites, particular emphasis is needed to control the different chemical compounds synthesized by them. Moreover, the Bacillus and Trichoderma spp. display different preferences with respect to growth rate, morphology and culture conditions. It would, therefore be very convenient to establish a suitable liquid fermentation process to enable the co-culture of the above two organisms in order to improve the resistance to pathogens, along with harvesting novel chemical compounds during cultivation. It has been reported that a potent anticancerous drug paclitaxel can be produced by the yew tree fungal endophyte Paraconiothyrium. Interestingly, when co-cultivated with other fungi such as Alternaria sp. or Phomopsis sp., the titre of this alkaloid was seen to increase [13]. Similarly, co-culture of fungus Aspergillus nidulans challenged by Streptomyces rapamycinicus provided the cue for the molecular basis of induction of silent fungal biosynthetic gene clusters $[14,15]$. Thus, cocultivation methods have been developed and widely applied in the research of microbial secondary metabolites [16].

In this study, the liquid co-cultivation of Bacillus amyloliquefaciens ACCC11060 and Trichoderma asperellum GDFS1009 was attempted for the first time to harvest their individual advantages such as enhanced antibacterial effect or the stimulated generation of biochemicals. Through LC-MS/MS technology, the similarities and the differences among the antibacterial substances in the co-cultured and individually-cultured fermentation liquors were detected.

\section{Results and discussion}

The morphology of singly-cultured and co-cultured

B. amyloliquefaciens ACCC1 1060 and/or T. asperellum GDFS1009 fermentation liquors and their effects on controlling $B$. cinerea

Bacillus spp. are a type of bio-control bacteria explored for various large-scale applications with the principal mechanism of antibiosis. Trichoderma spp. are a similar bio-control fungi widely used for multiple antimicrobial mechanisms including competition and mycoparasitism.

In this study, the liquid co-cultivation of $B$. amyloliquefaciens ACCC11060 and T. asperellum GDFS1009 was attempted for the first time. It was observed that 3 days after their respective single-cultivations, the fermentation liquor B of B. amyloliquefaciens ACCC11060 appeared creamy white, while the color of fermentation liquor T of T. asperellum GDFS1009 was relatively lighter and appeared beige, with a uniform distribution of mycelia with low viscosity. However, when the inoculation ratio of B. amyloliquefaciens ACCC11060 and T. asperellum GDFS1009 was 1:1, the fermentation liquor BT1 was bright yellow after 3 days of co-cultivation. The mycelia of T. asperellum GDFS1009 were dominant, but they were significantly rarefied and the viscosity was high. Further, when the inoculation ratio of $B$. amyloliquefaciens ACCC11060 and T. asperellum GDFS1009 was 1.9:1, the fermentation liquor BT2 was creamy white after 3 days of co-cultivation. The Bacillus was found to be dominant, with few T. asperellum GDFS1009 mycelia with viscosity assembled together (Table 1).

While determining the antibacterial effect of fermentation liquor on B. cinerea, it was found that the co-cultured fermentation liquor BT1 exhibited a higher activity $(47.86 \pm 0.51 \%)$ as compared to singly-cultured fermentation liquor $\mathrm{T}(30.67 \pm 0.95 \%)$, but was lower than $\mathrm{B}$ $(58.89 \pm 1.34 \%)$. Interestingly, the effect of co-cultured fermentation liquor BT2 $(66.86 \pm 2.14 \%)$ was higher than both $\mathrm{B}$ and $\mathrm{T}$ (Fig. 1 and Table 1).

Table 1 Forms and inhibition rates of the fermentation solutions from pure-cultivation and co-cultivation of $B$. amyloliquefaciens ACCC1 1060 and T. asperellum GDFS1009

\begin{tabular}{|c|c|c|c|c|}
\hline \multirow[t]{2}{*}{ Name } & \multicolumn{2}{|l|}{ Inoculation quantity } & \multirow[t]{2}{*}{ Fermentation form } & \multirow[t]{2}{*}{ Inhibition rate (\%) } \\
\hline & $\begin{array}{l}\text { B. amyloliquefaciens } \\
\text { ACCC11060 (mL) }\end{array}$ & $\begin{array}{l}\text { T. asperellum } \\
\text { GDFS1009 (mL) }\end{array}$ & & \\
\hline B & 1 & 0 & Off-white suspension & $58.89 \pm 1.34$ \\
\hline $\mathrm{T}$ & 0 & 1 & Beige solution with loose mycelium & $30.67 \pm 0.95$ \\
\hline BT1 & 1 & 1 & Bright yellow solution with high-viscosity mycelia & $47.86 \pm 0.51$ \\
\hline BT2 & 1.9 & 0.1 & $\begin{array}{l}\text { Off-white suspension with a small amount of high- } \\
\text { viscosity mycelium }\end{array}$ & $66.86 \pm 2.14$ \\
\hline
\end{tabular}




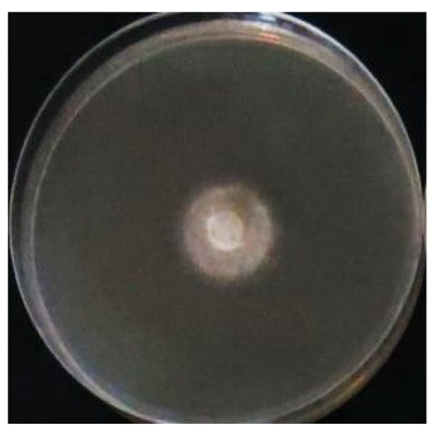

B

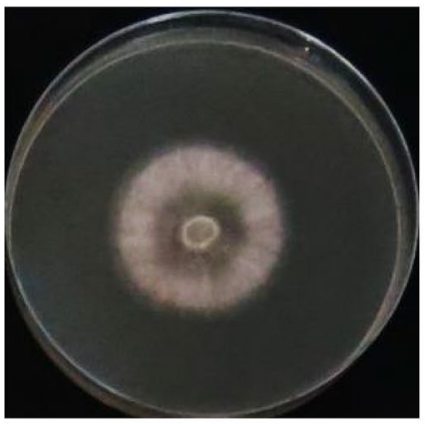

T

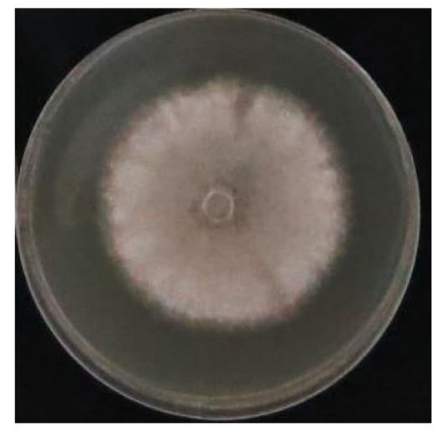

CK

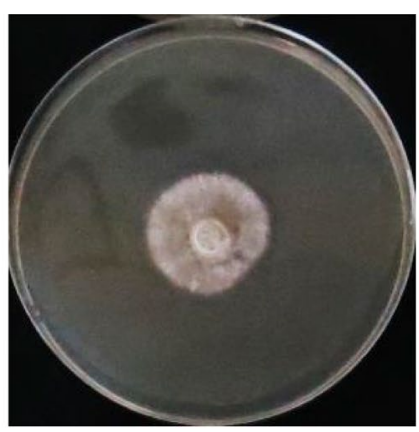

BT1

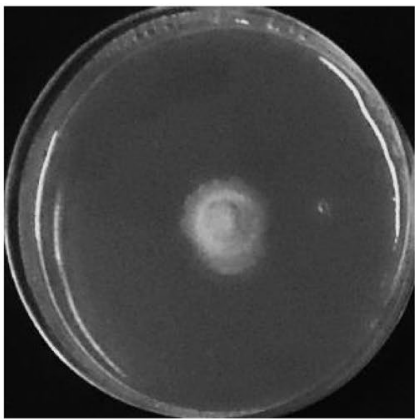

BT2

Fig. 1 Inhibition effects on B. cinerea and changed metabolites of the fermentation solutions

All the four fermentation liquors-B, T, BT1 and BT2 were subjected to intensive investigations.

The variance of analysis between fermentation liquor groups of singly-cultured and co-cultured $B$. amyloliquefaciens ACCC11060 and/or T. asperellum GDFS1009

After the OPLS-DA analysis of metabolites determined through LC-MS $(+)$ and LC-MS (-), it was found that there were significant differences in the metabolic compositions of B, T and BT1 fermentation liquors. However there were non-significant differences identified between BT2 and B, although both showed certain differences (Fig. 2). This was consistent with PCA analysis (data not shown).

Through LC-MS/MS analysis in the present study, 102 types were successfully annotated among the plethora of various metabolites. When the fermentation liquors $\mathrm{T}$ of $T$. asperellum GDFS1009 was compared with B of B. amyloliquefaciens ACCC11060, a total of 78 chemical compounds were found to be present with significant differences, with an elevation of 47 and 31 compounds in $\mathrm{B}$ and $\mathrm{T}$ respectively. The chemical compounds with significant variations included amino acids, organic acids, alcohols, aldehydes, flavonoids and alkaloids (Fig. 3).

\section{Analysis of differential compounds in singly-cultured and co-cultured B. amyloliquefaciens ACCC11060 and/or T. asperellum GDFS1009 fermentation liquors through LC- MS/MS}

Through the LC-MS/MS analysis, 76 compounds were found to exist with significant differences in a comprehensive comparison between B, T, BT1 and BT2 (Additional files 1,2). A total of 22 compounds were identified with significantly high proportion in $\mathrm{B}, 14$ were found in $\mathrm{T}$, while another 24 and 16 were found in BT1 and BT2 respectively (Fig. 4a).

L-Amino acids have various applications in food and feed biotechnology and they also serve as intermediates in chemical industry [17]. With the positive growth of the amino acid market, the microbial production of amino acids is drawing more attention. Interestingly, in this study, among the compounds with significant variations, it was noticed that amino acids accounted for $23.68 \% .3$ amino acids were found significantly high in B and included L-lysine, L-tryptophan and L-tyrosine. Similarly, 3 amino acids found significantly high in $\mathrm{T}$ included 

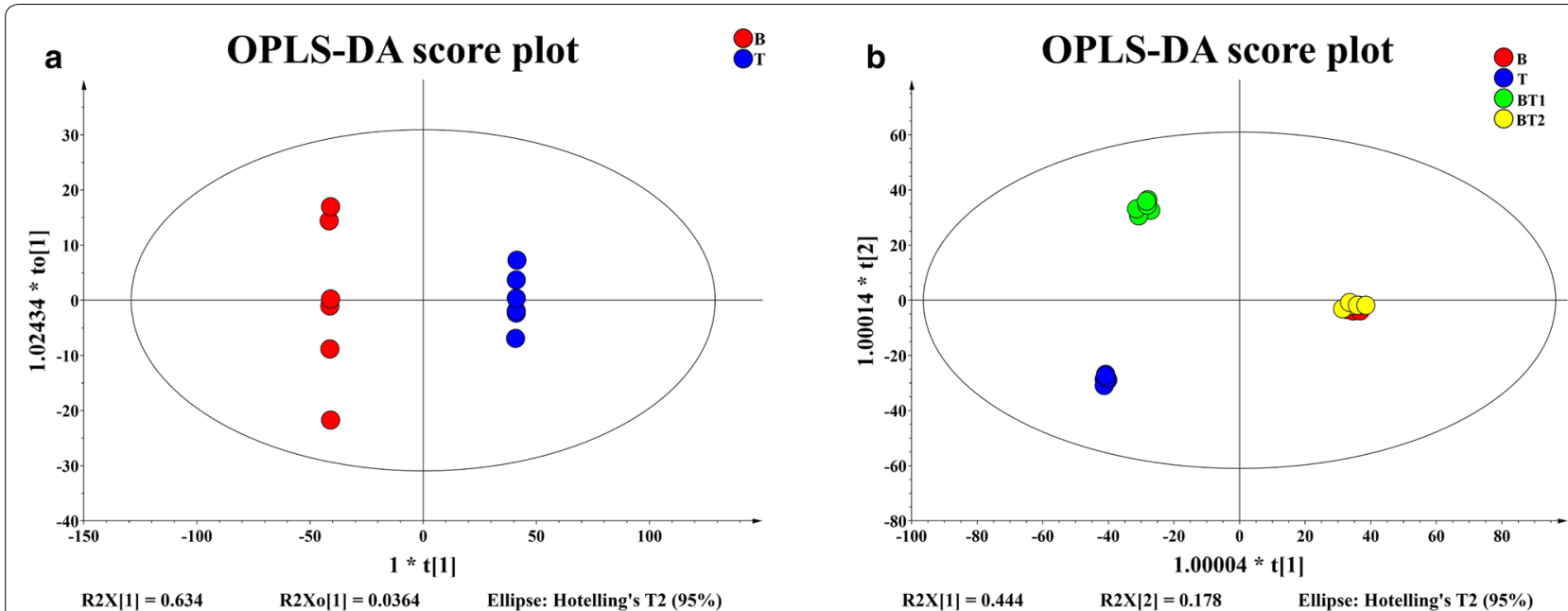

Fig. 2 OPLS-DA analysis of the metabolism differences among the fermentation solutions from pure-cultivation and co-cultivation of $B$. amyloliquefaciens ACCC1 1060 and T. asperellum GDFS1009 based on LC-MS. a Differences of the pure-cultivations; $\mathbf{b}$ differences among the pure-cultivations and co-cultivations

5-oxo-L-proline, L-asparagine and L-aspartic acid. There were a total of 8 amino acids found in significantly high proportions in BT1 and included D-aspartic acid, L-allothreonine, L-glutamic acid, L-histidine, L-isoleucine, L-leucine, L-proline and L-serine. Lastly, 4 amino acids identified with significant high yields in BT2 included D-phenylalanine, L-kynurenine, $N$-acetyl-L-glutamate and $N$-acetyl-L-leucine (Table 2). Among these, many rare amino acids were found including 5-oxo-L-proline in $\mathrm{B}, \mathrm{L}$-allothreonine and L-glutamic acid in BT1, and L-kynurenine, $N$-acetyl-L-glutamate and $N$-acetyl-L-leucine in BT2. Taken together, these data provide an excellent theoretical guidance for large-scale production of specific amino acids in the industry.

Among the compounds with significant variations, the substances related to biological control accounted to about $19.73 \%$. There were a total of 4 antimicrobial substances identified in B with significantly high proportions and they included 2-aminoisobutyric acid, 5-aminolevulinic acid, dibutyl phthalate and gatifloxacin. Citric acid was found in abundance in T. Similarly, 5 antibacterial substances with significantly high yield were present in BT1 and included 4-hydroxybenzoic acid, apigenin, glycine betaine, malic acid and nicotinic acid, while another 5 antibacterial substances identified with significantly high yield in BT2 were indole-3-acetic acid, indole-3-carboxylic acid, phenacylamine, trans-3-coumaric acid and trans-cinnamic acid. Among these compounds, some inhibited fungus and some inhibited bacteria, while some displayed the additional functions of expelling insects or regulating plant growth (Table 3 and Fig. 4b). For example, the dibutyl phthalate abundantly present in B could potentially inhibit Rhizoctonia solani [18]. Likewise, citric acid, showing a high yield in T could effectively inhibit Shigella species [19]. Both glycine betaine and malic acid, present in high proportions in BT1 could effectively inhibit Fusarium sp. [20, 21]. Similarly, the indole-3-acetic acid with an elevated presence in BT2 could effectively inhibit Fusarium solani f. sp. Eumartii [22]. Some compounds identified were priorly proven for their antibacterial functions. For example, the 4-hydroxybenzoic acid with a significantly high yield in BT1 could effectively inhibit Staphylococcus aureus [23]. The trans-cinnamic acid with a significantly high yield in BT2 could inhibit E. coli, Listeria etc. [24]. For different types of plant diseases, the following targeted controls were conducted by selecting singly-cultured or co-culture models, and a certain specific compound could be developed through chemical separation method to prevent and control a specific disease. In addition, some compounds displayed other functions. For example, the 5-aminolevulinic acid with a high yield in B was capable of expelling insects and weeding. The indole-3-acetic acid with a high yield in BT2 could promote plant growth [25]. This establishes a foundation for insect-resistance and growth promotion for further applications in agriculture.

In the current study, many differential compounds were determined through methanol water extraction alone, aided with LC-MS/MS technology. It is possible to generate additional differential antibacterial compounds through other extraction and determination methods. By employing UPLC and NMR technologies, the unknown 


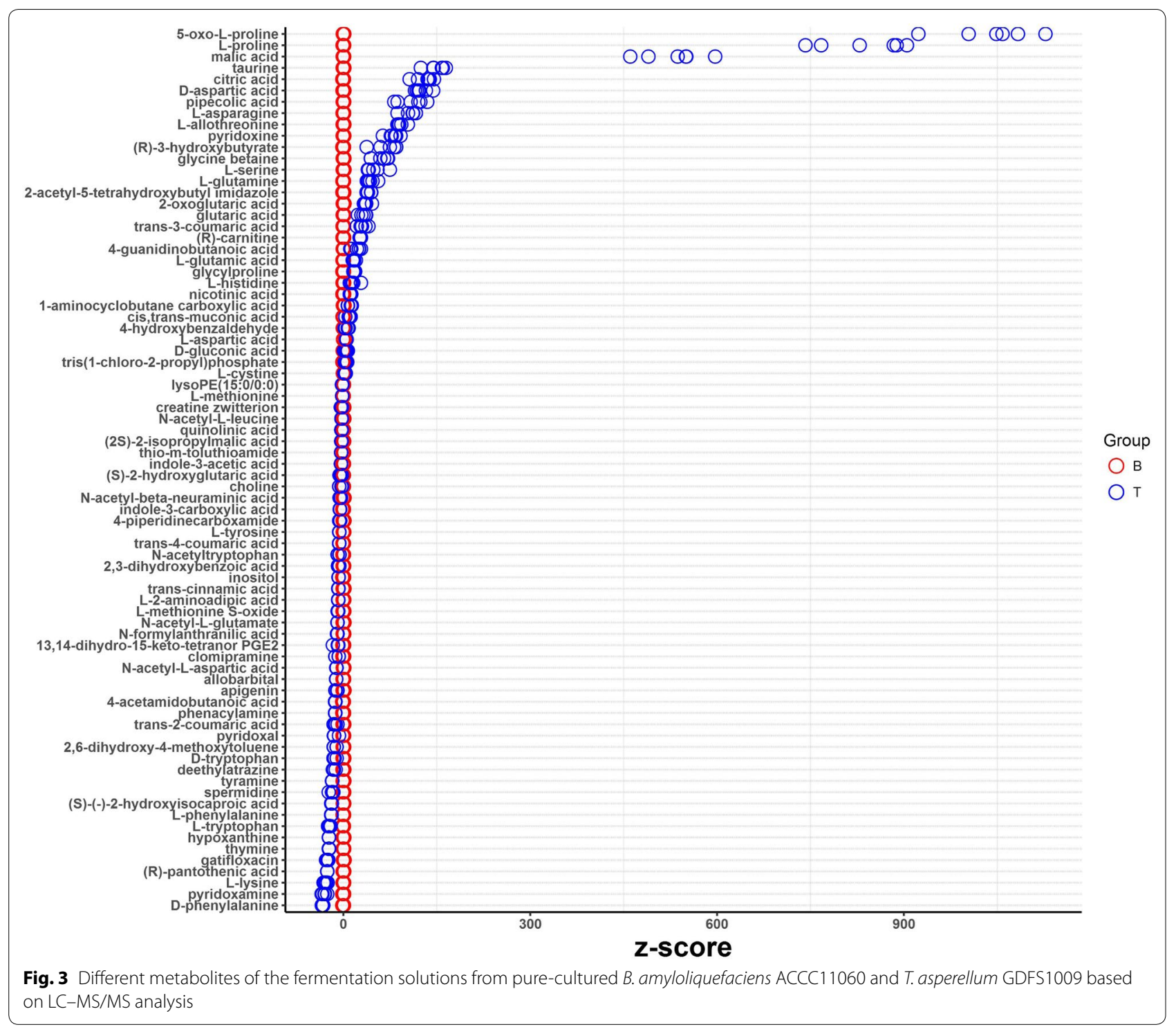

antibacterial compounds could also be isolated for identification and the modification types and new skeletons of novel compounds could be unraveled.

\section{The KEGG analysis of differential compounds} in singly-cultured and co-cultured B. amyloliquefaciens ACCC1 1060 and/or T. asperellum GDFS1009 fermentation liquors

A KEGG analysis was conducted with the 76 differential metabolites generated from the comprehensive comparison between B, T, BT1 and BT2. The integrative calculation of impact and $-\log (\mathrm{p})$ indicated that the 76 metabolites were involved in a total of 78 metabolic pathways (Fig. 5).
According to the rank of Impact numerical values, it was found that in 15 metabolic pathways with the most significant variations, 9 were related with amino acid metabolism such as the alanine, aspartate and glutamate metabolism, D-glutamine and D-glutamate metabolism, histidine metabolism, tryptophan metabolism, arginine and proline metabolism, lysine degradation, lysine biosynthesis, biosynthesis of amino acids, glycine, serine and threonine metabolism. The other five were taurine and hypotaurine metabolism, 2-oxocarboxylic acid metabolism, vitamin B6 metabolism, aminobenzoate degradation, tropane, piperidine and pyridine alkaloid biosynthesis, and citrate cycle (Table 4). Additionally, the metabolic pathways 


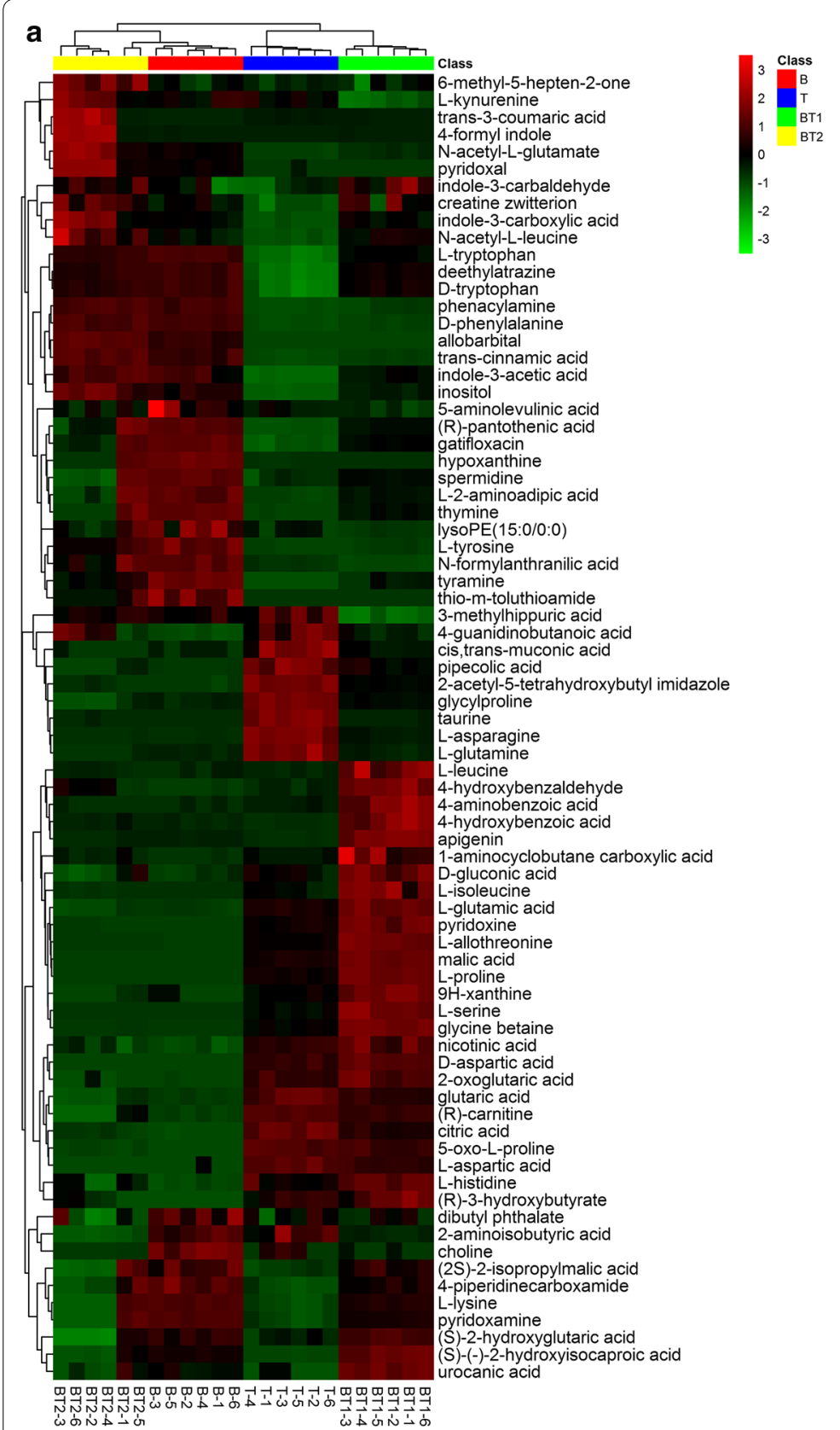<smiles>NCC(=O)CCC(=O)O</smiles>

5-aminolevulinic acid

C<smiles>CCC(O)(O)C(O)(O)c1cnc(C(C)=O)[nH]1</smiles>

2-acetyl-5-tetrahydroxybutyl imidazole

d<smiles>O=c1cc(-c2ccc(O)cc2)oc2cc(O)cc(O)c12</smiles>

e

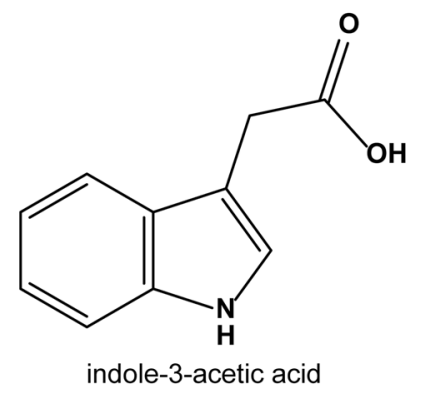

Fig. 4 Significantly changed metabolites among the fermentation solutions from pure-cultivation and co-cultivation of B. amyloliquefaciens ACCC1 1060 and T. asperellum GDFS1009 based on LC-MS/MS. a Global analysis with Heatmap; b the metabolite that are only highly productive in $\mathrm{B} ; \mathbf{c}$ the metabolite that are only highly productive in $\mathrm{T}$; $\mathbf{d}$ the metabolite that are only highly productive in BT1; e the metabolite that are only highly productive in BT2

in which the differential compounds were involved included indole alkaloid biosynthesis, flavone and flavonol biosynthesis, flavonoid biosynthesis, biosynthesis of antibiotics etc. (data not shown).
Correlation analysis on the differential compounds in singly-cultured and co-cultured B. amyloliquefaciens ACCC1 1060 and/or T. asperellum GDFS1009 fermentation liquors

The importance of correlation analysis is to find the association between different compounds. In 
Table 2 Amino acid with high yield under only one culture condition

\begin{tabular}{llll}
\hline Compound & VIP & p-value & $\begin{array}{l}\text { Culture } \\
\text { condition }\end{array}$ \\
\hline D-Tryptophan & 1.10083 & $2.63996 \mathrm{E}-14$ & $\mathrm{~B}$ \\
L-Lysine & 1.27053 & $3.31824 \mathrm{E}-05$ & $\mathrm{~B}$ \\
L-Tryptophan & 1.34496 & $3.59313 \mathrm{E}-18$ & $\mathrm{~B}$ \\
L-Tyrosine & 1.11095 & $1.33916 \mathrm{E}-12$ & $\mathrm{~B}$ \\
5-Oxo-L-proline & 1.02467 & $5.02938 \mathrm{E}-19$ & $\mathrm{~T}$ \\
L-Asparagine & 1.35007 & $1.32867 \mathrm{E}-18$ & $\mathrm{~T}$ \\
L-Aspartic acid & 1.00148 & $5.98419 \mathrm{E}-13$ & T \\
D-Aspartic acid & 1.25926 & $1.18975 \mathrm{E}-20$ & BT1 \\
L-Allothreonine & 1.11358 & $3.55869 \mathrm{E}-21$ & BT1 \\
L-Glutamic acid & 1.30872 & $2.87953 \mathrm{E}-17$ & BT1 \\
L-Histidine & 1.09861 & $1.18916 \mathrm{E}-07$ & BT1 \\
L-Isoleucine & 1.09781 & $6.80041 \mathrm{E}-10$ & BT1 \\
L-Leucine & 1.26627 & $7.57847 \mathrm{E}-10$ & BT1 \\
L-Proline & 1.10174 & $3.35974 \mathrm{E}-23$ & BT1 \\
L-Serine & 1.14096 & $5.7145 \mathrm{E}-16$ & BT1 \\
D-Phenylalanine & 1.02392 & $9.58885 \mathrm{E}-21$ & BT2 \\
L-Kynurenine & 1.10698 & $2.25127 \mathrm{E}-06$ & BT2 \\
N-Acetyl-L-glutamate & 1.31966 & $6.89686 \mathrm{E}-08$ & BT2 \\
N-Acetyl-L-leucine & 1.20319 & $1.76547 \mathrm{E}-06$ & BT2 \\
\hline
\end{tabular}

production, the yield of one compound can be increased by increasing the amount of another compound.

A correlation analysis on the 76 differential metabolites generated through the comprehensive comparison between B, T, BT1 and BT2 was conducted.

A positive correlation was present among D-aspartic acid, L-allothreonine, L-glutamic acid, L-isoleucine,
L-proline and L-serine which had high yields in BT1. While the $\mathrm{L}$-aspartic acid which displayed a high yield in $\mathrm{T}$ presented a positive correlation with them, $\mathrm{L}$-lysine with high yield in B had no correlation with them at all. Further, L-kynurenine with a high yield in BT2 posed a negative correlation with them. In addition, the citric acid with a high yield in $\mathrm{T}$ displayed a positive correlation with D-aspartic acid, L-allothreonine, L-glutamic acid, L-isoleucine, L-proline and L-serine.

The 5-aminolevulinic acid which exhibited a high yield in B had no correlation with 2-acetyl-5-tetrahydroxybutyl imidazole which had a high yield in T. Similarly, the apigenin with a high yield in BT1 displayed no correlation with indole-3-acetic acid which had a high yield in BT2 (Fig. 6).

This information provides a technical guidance for generating a combination of compounds for use in agriculture or industry. During large scale production of compounds, the same observation may be considered for the purpose of increasing the generation of amino acids by increasing the ratio of citric acid in the medium.

\section{Conclusions}

When an inoculation proportion between B. amyloliquefaciens ACCC11060 and T. asperellum GDFS1009 is 1.9:1, the antimicrobial effects of the resulting co-cultured fermentation liquor is significantly higher than those of single cultivations. The syntheses of some antimicrobial substances contributed to the observed increase. In addition, when the inoculation proportion between B. amyloliquefaciens ACCC11060 and T. asperellum

Table 3 Bio-control related metabolites with highly productive under only one culture condition

\begin{tabular}{|c|c|c|c|c|}
\hline Compound & VIP & p-value & Culture condition & Function \\
\hline 2-Aminoisobutyric acid & 1.36007 & $1.74 \mathrm{E}-07$ & $\mathrm{~B}$ & Candida albicans [35] \\
\hline 5-Aminolevulinic acid & 1.00584 & 0.002082456 & B & Herbicide/insecticide [36] \\
\hline Dibutyl phthalate & 1.05246 & 0.007555153 & $\mathrm{~B}$ & Rhizoctonia solani [37] \\
\hline Gatifloxacin & 1.17378 & $1.15 E-07$ & $\mathrm{~B}$ & Staphylococcus aureus [38] \\
\hline Citric acid & 1.26357 & $1.65 E-16$ & $\mathrm{~T}$ & Shigella sp. $[19,39]$ \\
\hline 4-Hydroxybenzoic acid & 1.32557 & $2.41 \mathrm{E}-12$ & BT1 & Staphylococcus aureus, Fusarium culmorum [23] \\
\hline Apigenin & 1.35917 & $2.34 \mathrm{E}-18$ & BT1 & Alternaria tenuissima, Salmonella typhimurium $[40,41]$ \\
\hline Glycine betaine & 1.13412 & $3.00 \mathrm{E}-20$ & BT1 & Fusarium verticillioides [20] \\
\hline Malic acid & 1.3502 & 8.97E-23 & BT1 & Aspergillus flavus, Fusarium oxysporum [21] \\
\hline Nicotinic acid & 1.01745 & $1.01 \mathrm{E}-10$ & BT1 & Staphylococcus aureus, Aspergillus niger $[42,43]$ \\
\hline Indole-3-acetic acid & 1.34315 & $6.82 \mathrm{E}-11$ & BT2 & Fusarium solani f. sp. eumartii [22] \\
\hline Indole-3-carboxylic acid & 1.31271 & $4.53 \mathrm{E}-06$ & $\mathrm{BT} 2$ & Plectosphaerella cucumerina [44] \\
\hline Phenacylamine & 1.01991 & $3.66 \mathrm{E}-19$ & BT2 & Bactria $[45,46]$ \\
\hline Trans-3-coumaric acid & 1.13656 & 0.000448228 & BT2 & Escherichia coli, Staphylococcus aureus [47] \\
\hline Trans-cinnamic acid & 1.03921 & $4.05 E-18$ & BT2 & Fusicladium effusum, Listeria $[24,48]$ \\
\hline
\end{tabular}




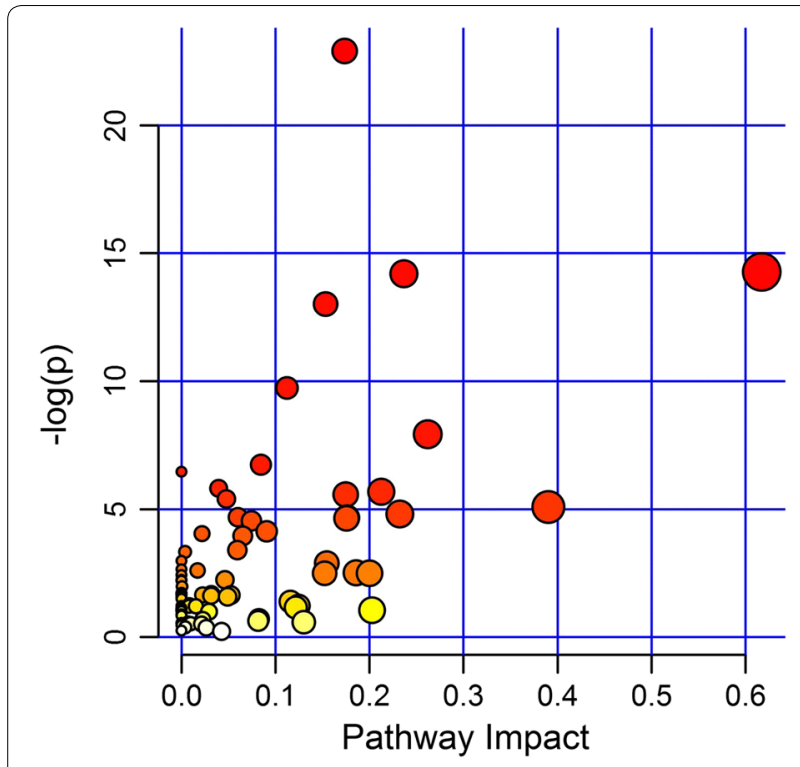

Fig. 5 KEGG pathway analysis of the changed metabolites

GDFS1009 is 1:1, the co-cultivation would enhance the production of specific amino acids. This could be further explored for a large scale production of amino acids or at least serve as a theoretical basis for the generation of certain rare amino acids.

\section{Methods}

\section{Strains}

The biocontrol bacterium $B$. amyloliquefaciens ACCC11060 was purchased from Agricultural Culture
Collection of China (ACCC NO. 11060), Beijing, China. The biocontrol fungus T. asperellum GDFS1009 was preserved in China General Microbiological Culture Collection Center (CGMCC NO. 9512), Beijing, China. $B$. cinerea on tomato was used as a target pathogen for the examination of biocontrol effects.

\section{The co-cultured combinations}

The liquid BP broth (Beef extract $0.3 \%$ and peptone $0.5 \%$ ) was used in this study for both pure and co-cultivation.

Trichoderma asperellum GDFS1009 was incubated at a constant temperature of $28{ }^{\circ} \mathrm{C}$ in PAD plates for 3 days, following which the spores were washed with sterile water, filtered through degreasing cotton, inoculated into BP broth to a final concentration of $10^{6} \mathrm{cfu} / \mathrm{mL}$, and cultured with shaking at $180 \mathrm{rpm}$ overnight at $28{ }^{\circ} \mathrm{C}$, until the $\mathrm{OD}_{600}$ reached 1.0. B. amyloliquefaciens ACCC11060 was incubated in LB culture dishes for 2 days, following which the cells were washed with sterile water, inoculated into BP broth, and cultured with shaking at $180 \mathrm{rpm}$ overnight at $28^{\circ} \mathrm{C}$ until the $\mathrm{OD}_{600}$ reached 1.0.

$1 \mathrm{~mL}$ of B. amyloliquefaciens ACCC11060 was singly inoculated into $100 \mathrm{~mL}$ BP broth, fermented at $180 \mathrm{rpm}$ and $28{ }^{\circ} \mathrm{C}$ for 3 days, and the resulting fermentation liquor was named as B. Similarly, $1 \mathrm{~mL}$ of $T$. asperellum GDFS1009 was singly inoculated into $100 \mathrm{~mL}$ BP broth, fermented at $180 \mathrm{rpm}$ and $28{ }^{\circ} \mathrm{C}$ for 3 days, and the resulting fermentation liquor was labelled as $\mathrm{T}$.

$1 \mathrm{~mL}$ each of B. amyloliquefaciens ACCC11060 and T. asperellum GDFS1009 in equal proportions were inoculated into $100 \mathrm{~mL} \mathrm{BP}$ broth, co-cultured at $180 \mathrm{rpm}$ and $28{ }^{\circ} \mathrm{C}$ for 3 days, and the resulting fermentation liquor

Table 4 Significantly changed KEGG pathways

\begin{tabular}{lcccc}
\hline Pathway & Total & Hits & -LOG(p) & Impact \\
\hline Alanine, aspartate and glutamate metabolism & 28 & 7 & 14.271 & 0.61709 \\
Taurine and hypotaurine metabolism & 20 & 3 & 5.0872 & 7.9343 \\
D-Glutamine and D-glutamate metabolism & 8 & 3 & 14.199 \\
2-Oxocarboxylic acid metabolism & 108 & 12 & 4.8140 \\
Vitamin B6 metabolism & 22 & 3 & 5.6843 & 0.2619 \\
Histidine metabolism & 33 & 4 & 1.0488 & 0.23654 \\
Aminobenzoate degradation & 64 & 2.4945 & 0.2322 \\
Tropane, piperidine and pyridine alkaloid biosynthesis & 25 & 2 & 2.5133 \\
Tryptophan metabolism & 53 & 3 & 4.6681 & 0.21248 \\
Arginine and proline metabolism & 68 & 5 & 4.6444 \\
Lysine degradation & 44 & 4 & 5.5736 \\
Lysine biosynthesis & 34 & 4 & 0.20265 \\
Biosynthesis of amino acids & 127 & 17 & 0.18578 \\
Citrate cycle (TCA cycle) & 20 & 2.916 & 0.17587 \\
Glycine, serine and threonine metabolism & 47 & 2.8901 & 0.17555 \\
\hline
\end{tabular}

Total, the total number of metabolites in the target metabolic pathway; Hits, the number of differential metabolites in the target metabolic pathway; - log (p): -log ( $p$-value); impact, the greater the effect of metabolic pathways, the better 


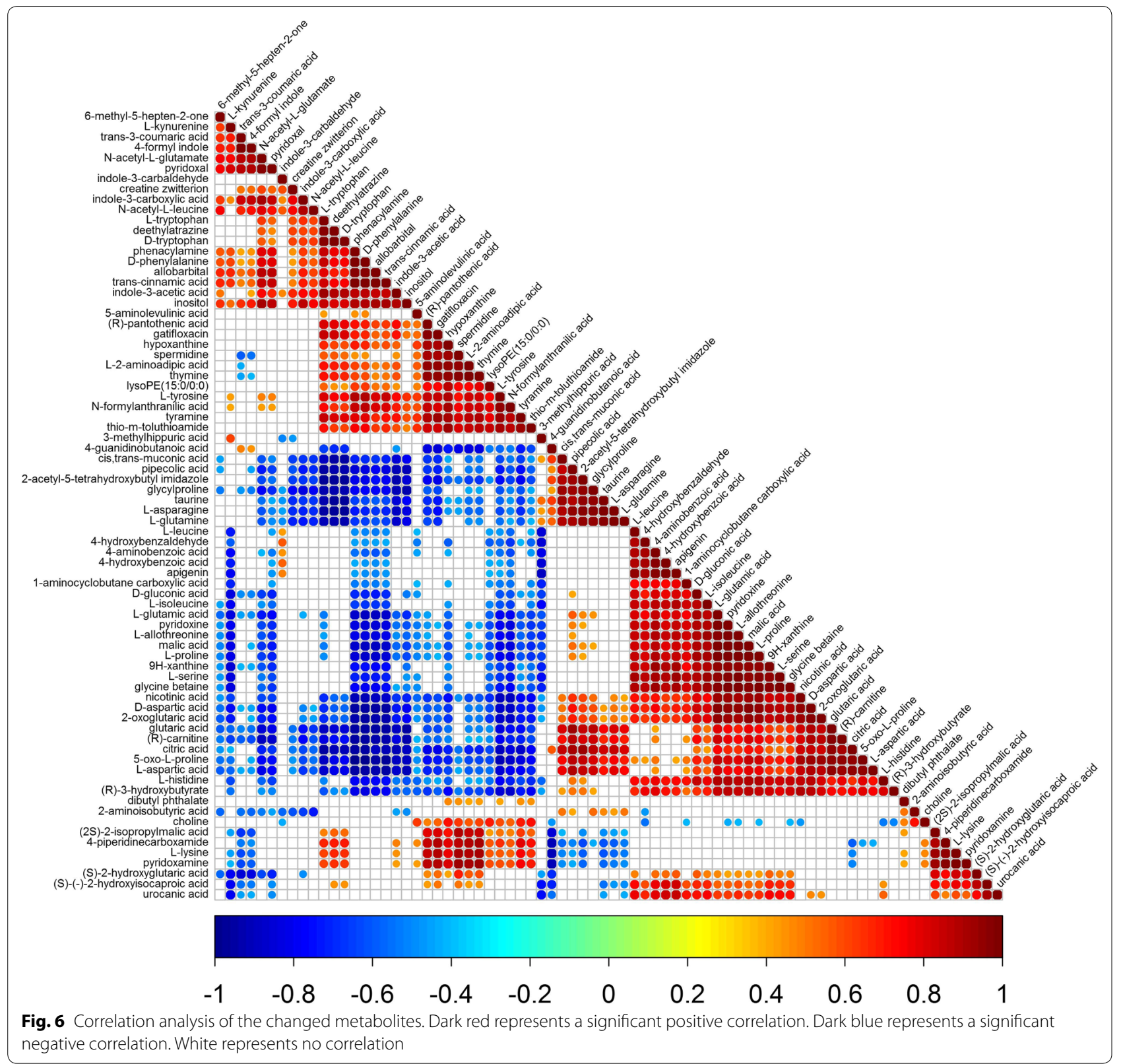

was named as BT1. Lastly, $1.9 \mathrm{~mL}$ of B. amyloliquefaciens ACCC11060 and $1 \mathrm{~mL}$ of T. asperellum GDFS1009 were inoculated into $100 \mathrm{~mL} \mathrm{BP}$ broth, co-cultured at $180 \mathrm{rpm}$ and $28{ }^{\circ} \mathrm{C}$ for 3 days, and the resulting fermentation liquor was labeled as BT2.

Apart from these, many other combination models were attempted with different inoculation ratios of Trichoderma sp. and Bacillus sp. As they were not typical (Neither natural trial option nor best inhibition on $B$. cinerea), we didn't descript it for details. Each group of experiments had three repeats.

\section{Antibacterial effects}

Botrytis cinerea was incubated at a constant temperature of $25^{\circ} \mathrm{C}$ in PAD culture dishes for 5 days, and the fungus cake was passed through a $7 \mathrm{~mm}$ hole punch. $10 \mathrm{~mL}$ each of the above multiple groups of co-cultured and singly-cultured fermentation liquors, along with $10 \mathrm{~mL}$ of blank BP broth serving as control, were filtered through Millipore filters of pore size $0.22 \mu \mathrm{m}$ and added into $40 \mathrm{~mL}$ of PDA broth at a temperature of $55^{\circ} \mathrm{C}$, following which the contents were mixed well and poured into plates. The fungus cakes of $B$. cinerea were then inversely placed in the middle of each plate and incubated at a constant temperature 
of $25^{\circ} \mathrm{C}$ for 3 days. The colony diameters were determined by applying the 'crossing method' and photographed. Each group of experiments had three repeats.

\section{Preparation of samples for LC-MS/MS}

$50 \mathrm{~mL}$ each of the fermentation liquors B, T, BT1 and BT2, along with $50 \mathrm{~mL}$ of blank BP broth serving as control, were filtered through Millipore filters of pore size $0.22 \mu \mathrm{m}$ and snap-frozen with liquid nitrogen, following which they were transported on dry ice to Suzhou BioNovoGene Metabolomics Platform.

All the samples were thawed at $4{ }^{\circ} \mathrm{C}$ and mixed uniformly. $200 \mu \mathrm{L}$ of each sample was taken in a $1.5 \mathrm{~mL}$ microcentrifuge tube, to which $800 \mu \mathrm{L}$ of methyl alcohol was added and vibrated for $60 \mathrm{~s}$ to mix the contents uniformly, following which the tubes were centrifuged at 12,000 rpm at $4{ }^{\circ} \mathrm{C}$ for $10 \mathrm{~min}$. The supernatants were transferred to a new $1.5 \mathrm{~mL}$ microcentrifuge tube, concentrated and dried by vacuum and re-dissolved in $300 \mu \mathrm{L}$ of $80 \%$ methyl alcohol. Next, the supernatants were filtered through a $0.22 \mu \mathrm{m}$ membrane to obtain the samples for determination. $20 \mu \mathrm{L}$ of each sample was kept aside for QC, while the remaining was analyzed through LC-MS [26, 27].

For the chromatographic study, Waters ACQUITY UPLC instrument was used with ACQUITY UPLC ${ }^{\circledR} B E H$ C18 $1.7 \mu \mathrm{m}(2.1 \times 100 \mathrm{~mm})$ chromatographic column. The temperature of automatic sampler was set to $4{ }^{\circ} \mathrm{C}$ with a flow velocity of $0.25 \mathrm{~mL} / \mathrm{min}$. The column temperature was $40{ }^{\circ} \mathrm{C} .10 \mu \mathrm{L}$ of sample was introduced for gradient elution, and the mobile phase was $0.1 \%$ formic acid water (A) $-0.1 \%$ formic acid acetonitrile (B). The gradient elution program was as follows: $0-1 \mathrm{~min}, 2 \% \mathrm{~B}$; 1-9.5 min, 2-50\% B; 9.5-14 min, 50-98\% B; 14-15 min, 98\% B; 15-15.5 min, 98-2\% B; 15.5-17 min, 2\% [28].

For the mass spectrometry study, the Thermo LTQ Orbitrap XL instrument was used with electrospray ionization (ESI) and cation-anion ionization mode. The voltages for positive and negative ion sprays were $4.80 \mathrm{kV}$ and $4.50 \mathrm{kV}$ respectively. The sheath gas was $45 \mathrm{arb}$ and auxiliary gas was 15 arb. The capillary temperature was $325^{\circ} \mathrm{C}$, and the capillary voltage was $35 \mathrm{~V} /-15 \mathrm{~V}$. The voltage of tube lens was $50 \mathrm{~V} /-50 \mathrm{~V}$ and the full scan was conducted with the resolution of 60,000 , with the scan scope being 89-1000. CID was applied for secondary dissociation with a fragmentor voltage of $30 \mathrm{eV}$. Simultaneously, dynamic exclusion (with the repeat count of 2) was applied to eliminate the unnecessary MS/MS information, with the time for dynamic exclusion set as $15 \mathrm{~s}$ [29].

\section{Analysis of metabolome data}

The data pre-processing included the following: (a) the original data obtained was converted into mzXML format (xcms input file format) using Proteowizard software (v3.0.8789) [30]. (b) The XCMS software package of $\mathrm{R}$ (v3.3.2) was applied for peaks identification, filtration and alignment. The major parameters included $\mathrm{bw}=5$, $\mathrm{ppm}=15, \quad$ peakwidth $=\mathrm{c} \quad(10,120), \quad$ mzwid $=0.015$, mzdiff $=0.01$ and method $=$ "centWave" [31]. (c) The data matrix containing the information of mass to charge ratio $(\mathrm{m} / \mathrm{z})$, retention time and peak area (intensity) was obtained. The positive and negative ion models gathered 3680 and 3566 precursor molecules respectively. The data were collected and the following analyses were conducted. (d) For the comparison between data of different orders, the batch normalization of peak area was conducted.

Few multivariate statistical analyses including principal component analysis (PCA) and Orthogonal Projections to Latent Structures Discriminant Analysis (OPLS-DA) were conducted to reveal the differences in the metabolic compositions between different comparison groups [32].

The LIGAND database of KEGG included the information related to chemical substances, enzyme molecules and reactions, while the metabolic pathways which the chemical compounds with specific differences were involved in were analyzed through KEGG [33].

The correlations between the metabolites were analyzed by calculating the Pearson or Spearman Rank correlation coefficient between any two metabolites. When the linear relation between two metabolites increased, the correlation coefficient trended toward 1 or -1 i.e., it trended towards 1 during a positive correlation and -1 during a negative correlation, while the calculation method was the cor() function in R (v3.1.3). The correlation analyses of metabolites and statistical test for significance were conducted simultaneously and the statistical test method was the cor.test() function in $\mathrm{R}$ language package. In addition, the false positive check on p-value was conducted and FDR p-value $\leq 0.05$ was used as the significant correlation [34].

\section{Additional files}

Additional file 1. Categories of all annotated compounds based on LC-MS/MS.

Additional file 2. Details of all annotated compounds based on LC-MS/ MS.

\section{Authors' contributions}

Conceived the study: JC. Generated the data: QW, MN, and KD. Performed the analyses: QW and MN. Made substantial contributions to acquisition of data: KD, JT, JR and CY. Wrote and polished the manuscript: QW, MN and JC. All authors read and approved the final manuscript. 


\begin{abstract}
Author details
${ }^{1}$ School of Agriculture and Biology, Shanghai Jiao Tong University, Shanghai 200240, China. ${ }^{2}$ Anhui Province Key Laboratory of Embryo Development and Reproductive Regulation, Anhui Province Key Laboratory of Environmental Hormone and Reproduction, Fuyang Normal University, Fuyang 236037, Anhui, China. ${ }^{3}$ Suzhou BioNovoGene Metabolomics Platform, Suzhou 215000, China.
\end{abstract}

\section{Acknowledgements}

We sincerely thank Suzhou BioNovoGene Metabolomics Platform for the assistance in LC-MS/MS analysis.

\section{Competing interests}

The authors declare that they have no competing interests.

\section{Availability of data and materials}

All data generated during this study are included in this published article.

\section{Consent for publication}

Not applicable.

\section{Ethics approval and consent to participate}

Not applicable.

\section{Funding}

This work was supported by National Key Research and Development Program of China (2017YFD0200403), National Key Research and Development Program of China (2017YFD0200901), National Natural Science Foundation of China (Nos. 31270155, 31672072), Beijing Natural Science Foundation (No. 6152007), and Shanghai Agriculture Development Promoted by Science and Technology (2017-1-6), CARS-02.

\section{Publisher's Note}

Springer Nature remains neutral with regard to jurisdictional claims in published maps and institutional affiliations.

\section{Received: 5 July 2018 Accepted: 25 September 2018}

Published online: 03 October 2018

\section{References}

1. Beneduzi A, Ambrosini A, Passaglia LM. Plant growth-promoting rhizobacteria (PGPR): their potential as antagonists and biocontrol agents. Genet Mol Biol. 2012;35(4 suppl):1044-51.

2. Haidar R, Fermaud M, Calvogarrido C, Roudet J, Deschamps A. Modes of action for biological control of Botrytis cinerea by antagonistic bacteria. Phytopathol Mediterr. 2016;55(3):301-22.

3. On A, Wong F, Ko Q, Tweddell RJ, Antoun H, Avis TJ. Antifungal effects of compost tea microorganisms on tomato pathogens. Biol Control. 2015;80:63-9.

4. Toure $Y$, Ongena M, Jacques P, Guiro AT, Thonart P. Role of lipopeptides produced by Bacillus subtilis GA1 in the reduction of grey mould disease caused by Botrytis cinerea on apple. J Appl Microbiol. 2004;96(5):1151-60.

5. Liu Y, Chen Z, Ng TB, Zhang J, Zhou M, Song F, Lu F, Liu Y. Bacisubin, an antifungal protein with ribonuclease and hemagglutinating activities from Bacillus subtilis strain B-916. Peptides. 2007:28(3):553-9.

6. Chakraborty K, Thilakan B, Raola VK. Antimicrobial polyketide furanoterpenoids from seaweed-associated heterotrophic bacterium Bacillus subtilis MTCC 10403. Phytochemistry. 2017;142:112-25.

7. Wise C, Novitsky L, Tsopmo A, Avis TJ. Production and antimicrobial activity of 3-hydroxypropionaldehyde from Bacillus subtilis strain CU12. J Chem Ecol. 2012;38(12):1521-7.

8. Harman GE. Overview of mechanisms and uses of Trichoderma spp. Phytopathology. 2006;96(2):190-4.

9. Bailey BA, Bae H, Strem MD, Crozier J, Thomas SE, Samuels GJ, Vinyard BT, Holmes KA. Antibiosis, mycoparasitism, and colonization success for endophytic Trichoderma isolates with biological control potential in Theobroma cacao. Biol Control. 2008;46(1):24-35.
10. Sharon E, Chet I, Viterbo A, Bareyal M, Nagan H, Samuels GJ, Spiegel Y. Parasitism of Trichoderma on Meloidogyne javanica and role of the gelatinous matrix. Eur J Plant Pathol. 2007:118(3):247-58.

11. Degenkolb T, Von Dohren H, Nielsen KF, Samuels GJ, Bruckner H. Recent advances and future prospects in peptaibiotics, hydrophobin, and mycotoxin research, and their importance for chemotaxonomy of Trichoderma and Hypocrea. Chem Biodivers. 2008;5(5):671-80.

12. Cutler HG, Cox RH, Crumley FG, Cole PD. 6-Pentyl-a-pyrone from Trichoderma harzianum: its plant growth inhibitory and antimicrobial properties. Agric Biol Chem. 1986;50(11):2943-5.

13. Soliman SSM, Raizada MN. Interactions between co-habitating fungi elicit synthesis of taxol from an endophytic fungus in host taxus plants. Front Microbiol. 2013:4:3.

14. Netzker T, Fischer J, Weber J, Mattern DJ, Konig CC, Valiante V, Schroeckh $\checkmark$, Brakhage AA. Microbial communication leading to the activation of silent fungal secondary metabolite gene clusters. Front Microbiol. 2015;6:299.

15. Marmann A, Aly AH, Lin W, Wang B, Proksch P. Co-cultivation-a powerful emerging tool for enhancing the chemical diversity of microorganisms. Mar Drugs. 2014:12(2):1043-65.

16. Sher D, Thompson JW, Kashtan N, Croal L, Chisholm SW. Response of Prochlorococcus ecotypes to co-culture with diverse marine bacteria. ISME J. 2011;5(7):1125-32.

17. Wendisch VF, Jorge JMP, Perez-Garcia F, Sgobba E. Updates on industrial production of amino acids using Corynebacterium glutamicum. World J Microbiol Biotechnol. 2016;32(6):10.

18. Ahsan T, Chen JG, Zhao XX, Irfan M, Wu YH. Extraction and identification of bioactive compounds (eicosane and dibutyl phthalate) produced by Streptomyces strain KX852460 for the biological control of Rhizoctonia solani AG-3 strain KX852461 to control target spot disease in tobacco leaf. AMB Express. 2017:7:1-9.

19. In Y, Kim J, Kim H, Oh S. Antimicrobial activities of acetic acid, citric acid and lactic acid against Shigella species. J Food Saf. 2013;33(1):79-85.

20. Dong Y, Dong K, Zheng Y, Tang L, Yang ZX. Faba bean fusarium wilt (Fusarium oxysporum) control and its mechanism in different wheat varieties and faba bean intercropping system. Ying Yong Sheng Tai Xue Bao. 2014;25(7):1979-87.

21. Schisler DA, Slininger PJ, Olsen NL. Appraisal of selected osmoprotectants and carriers for formulating Gram-negative biocontrol agents active against Fusarium dry rot on potatoes in storage. Biol Control. 2016:98:1-10.

22. Rania AB, Jabnoun-Khiareddine $H$, Nefzi $A$, Mokni-Tlili S, Daami-Remadi M Endophytic bacteria from Datura metel for plant growth promotion and bioprotection against Fusarium wilt in tomato. Biocontrol Sci Technol. 2016;26(8):1139-65

23. Cho JY, Moon JH, Seong KY, Park KH. Antimicrobial activity of 4-hydroxybenzoic acid and trans 4-hydroxycinnamic acid isolated and identified from rice hull. Biosci Biotechnol Biochem. 1998;62(11):2273-6.

24. Keawsa-ard S, Natakankitkul S, Liawruangrath S, Teerawutgulrag A, Trisuwan K, Charoenying P, Pyne SG, Liawruangrath B. Anticancer and antibacterial activities of the isolated compounds from Solanum spirale Roxb. leaves. Chiang Mai J Sci. 2012;39(3):445-54.

25. Ljung K, Östin A, Lioussanne L, Sandberg G. Developmental regulation of indole-3-acetic acid turnover in Scots pine seedlings. Plant Physiol. 2001;125(1):464-75.

26. Dunn WB, Broadhurst D, Begley P, Zelena E, Francismcintyre S, Anderson N, Brown M, Knowles JD, Halsall A, Haselden JN. Procedures for largescale metabolic profiling of serum and plasma using gas chromatography and liquid chromatography coupled to mass spectrometry. Nat Protoc. 2011;6(7):1060-83.

27. Zelena E, Dunn WB, Broadhurst D, Francismcintyre S, Carroll KM, Begley P, Ohagan S, Knowles JD, Halsall A, Wilson ID. Development of a robust and repeatable UPLC-MS method for the long-term metabolomic study of human serum. Anal Chem. 2009;81(4):1357-64.

28. Sangster T, Major H, Plumb RS, Wilson A, Wilson ID. A pragmatic and readily implemented quality control strategy for HPLC-MS and GC-MS-based metabonomic analysis. Analyst. 2006;131(10):1075-8.

29. Want EJ, Masson P, Michopoulos F, Wilson ID, Theodoridis G, Plumb RS, Shockcor JP, Loftus N, Holmes E, Nicholson JK. Global metabolic profiling of animal and human tissues via UPLC-MS. Nat Protoc. 2012;8(1):17-32. 
30. Smith CA, Want EJ, Omaille G, Abagyan R, Siuzdak G. XCMS: processing mass spectrometry data for metabolite profiling using nonlinear peak alignment, matching, and identification. Anal Chem. 2006;78(3):779-87.

31. Gatto L, Christoforou A. Using R and Bioconductor for proteomics data analysis. BBA. 2014;1844(1):42-51.

32. Thevenot EA, Roux A, Xu Y, Ezan E, Junot $C$. Analysis of the human adult urinary metabolome variations with age, body mass index, and gender by implementing a comprehensive workflow for univariate and OPLS statistical analyses. J Proteome Res. 2015;14(8):3322-35.

33. Kanehisa M, Sato Y, Kawashima M, Furumichi M, Tanabe M. KEGG as a reference resource for gene and protein annotation. Nucleic Acids Res. 2016;44:457-62

34. Rao G, Sui J, Zhang J. Metabolomics reveals significant variations in metabolites and correlations regarding the maturation of walnuts (Juglans regia L.). Biol Open. 2016;5(6):829-36.

35. Conlon JM, Al-Kharrge R, Ahmed E, Raza H, Galadari S, Condamine E. Effect of aminoisobutyric acid (Aib) substitutions on the antimicrobial and cytolytic activities of the frog skin peptide, temporin-1DRa. Peptides. 2007;28(10):2075-80.

36. Sasikala C, Ramana CV, Rao PR. 5-Aminolevulinic acid: a potential herbicide/insecticide from microorganisms. Biotechnol Prog. 1994;10(5):451-9.

37. Ahsan T, Chen J, Zhao X, Irfan M, Wu Y. Extraction and identification of bioactive compounds (eicosane and dibutyl phthalate) produced by Streptomyces strain KX852460 for the biological control of Rhizoctonia solani AG-3 strain KX852461 to control target spot disease in tobacco leaf. AMB Express. 2017;7(1):54

38. Fukuda $\mathrm{H}$, Hori S, Hiramatsu K. Antibacterial activity of gatifloxacin (AM-1155, CG5501, BMS-206584), a newly developed fluoroquinolone, against sequentially acquired Quinolone-resistant mutants and the norA transformant of Staphylococcus aureus. Antimicrob Agents Ch. 1998;42(8):1917-22.

39. Daly CG. Anti-bacterial effect of citric acid treatment of periodontally diseased root surfaces In vitro. J Clin Periodontol. 1982;9(5):386-92.
40. Liu R, Zhang H, Yuan M, Zhou J, Tu Q, Liu J, Wang J. Synthesis and biological evaluation of apigenin derivatives as antibacterial and antiproliferative agents. Molecules. 2013;18(9):11496-511.

41. Singh UP, Pandey VB, Singh KN, Singh RDN. Antifungal activity of some new fiavones and fiavone glycosides of Echinops echinatus. Botany. 1988;66(9):1901-3.

42. Naglah AM, Awad HM, Bhat MA, Alomar MA, Amr AEE. Microwaveassisted synthesis and antimicrobial activity of some novel isatin schiff bases linked to nicotinic acid via certain amino acid bridge. J Chem NY. 2015;2015:1-8.

43. Carlson LA. Nicotinic acid: the broad-spectrum lipid drug. A 50th anniversary review. J Intern Med. 2005;258(2):94-114.

44. Gamir J, Pastor V, Cerezo M, Flors V. Identification of indole-3-carboxylic acid as mediator of priming against Plectosphaerella cucumerina. Plant Physiol Biochem. 2012;61:169-79.

45. Bandyopadhaya A, Constantinou C, Psychogios N, Ueki R, Yasuhara S, Martyn JAJ, Wilhelmy J, Mindrinos MN, Rahme LG, Tzika AA. Bacterialexcreted small volatile molecule 2-aminoacetophenone induces oxidative stress and apoptosis in murine skeletal muscle. Int J Mol Med. 2016;37(4):867-78

46. Şirikci Gökhan AN, Gül Öztaş S, Gül Öztaş S. Synthesis, spectral, theoretical studies and in vitro antimicrobial activities of novel diphenyltin(IV) complexes of Schiff bases derived from phenacylamine. Appl Organomet Chem. 2014;28(7):537-44

47. Boz H. $p$-Coumaric acid in cereals: presence, antioxidant and antimicrobial effects. Int J Food Sci Technol. 2015;50(11):2323-8.

48. Bock CH, Shapiro-Ilan DI, Wedge DE, Cantrell CL. Identification of the antifungal compound, trans-cinnamic acid, produced by Photorhabdus luminescens, a potential biopesticide against pecan scab. J Pest Sci. 2013;87(1):155-62.
Ready to submit your research? Choose BMC and benefit from:

- fast, convenient online submission

- thorough peer review by experienced researchers in your field

- rapid publication on acceptance

- support for research data, including large and complex data types

- gold Open Access which fosters wider collaboration and increased citations

- maximum visibility for your research: over 100M website views per year

At BMC, research is always in progress.

Learn more biomedcentral.com/submissions 